\title{
The Power of Electron Diffraction Phase Analysis and Pattern Simulations Using the ICDD ${ }^{\circledR}$ Powder Diffraction File ${ }^{\mathrm{TM}}$ (PDF-4+)
}

\author{
Amy Gindhart ${ }^{1}$, Tom Blanton ${ }^{1}$, Justin Blanton ${ }^{1}$, Stacy Gates-Rector $^{1}$ \\ ${ }^{1 .}$ International Centre for Diffraction Data, Newtown Square, PA, USA
}

The Powder Diffraction File ${ }^{\mathrm{TM}}\left(\mathrm{PDF}^{\circledR}\right)$ is the only crystallographic database that is specifically designed for material identification and characterization. Materials included in the PDF products range from natural minerals and high-tech ceramics, to metals and alloys, polymers and pharmaceuticals. New developments in X-ray analysis and advances in scientific research have significantly influenced the range of data present in the PDF. ICDD's premier and most advanced database, PDF-4+, contains crystallographic and diffraction information used for phase identification of over 365,000 materials, as well as atomic coordinates for more than 251,000 phases. The PDF-4+ combines the world's largest sources of inorganic diffraction data from crystals and powders into a single database.

In addition to being a reference database used for the analysis of traditional single and multi-phase X-ray diffraction data, the PDF-4+ also supports electron diffraction (ED) based analyses. Often in materials characterization, X-ray, electron and neutron diffraction are used to complement one another. Given the complementary aspects of these techniques, it was logical to compile this data into one comprehensive database, the PDF-4+. As a result, a suite of electron diffraction based simulation tools have been designed. Research scientists often use electron diffraction as a method for obtaining higher diffraction intensities from nano-scale quantities of a material when X-ray scattering is limited. The increased intensity of an electron beam allows the user to investigate smaller samples and utilize a more focused beam to determine the atomic arrangement of crystals. However, the high energy beam can often promote multiple scattering interactions, making structure solution by electron diffraction alone relatively difficult compared with X-ray diffraction structure analyses. In support of the diffraction community, the ICDD has developed tools that enable users to specify a diffraction type (X-ray or electron) when using the PDF4+ reference database and its search/match capabilities.

The PDF-4+ currently has an electron diffraction tool suite that consists of simulated modules that generate selected area electron diffraction (SAED) patterns, electron backscatter diffraction (EBSD) patterns, 2-D ring patterns, and 1-D powder diffraction patterns. In addition, several data analysis features have been included that allow the user to obtain zone axis, spatial orientation, and crystallinity information. The simulations are derived using calculations based on the atomic parameters, electron scattering factors, and/or X-ray scattering factors specified in the PDF entry of interest [1]. Each simulation is interactive so the user can perform instantaneous alterations to the pattern by adjusting dynamic parameters such as zone axes, camera length, electron voltage, etc. The PDF-4+ software offers a variety of algorithms and simulation options that allow users to analyze electron diffraction data. In many cases, this provides a unique ability to analyze difficult material characterization problems. A new feature in PDF-4+ 2015 involves graphical overlaying of experimental spot patterns with calculated patterns from atomic coordinates. This allows the user to physically match their pattern with that of a known structure. This capability along with the other various electron diffraction simulation tools allows the PDF4+ to help identify phases using electron diffraction data. 


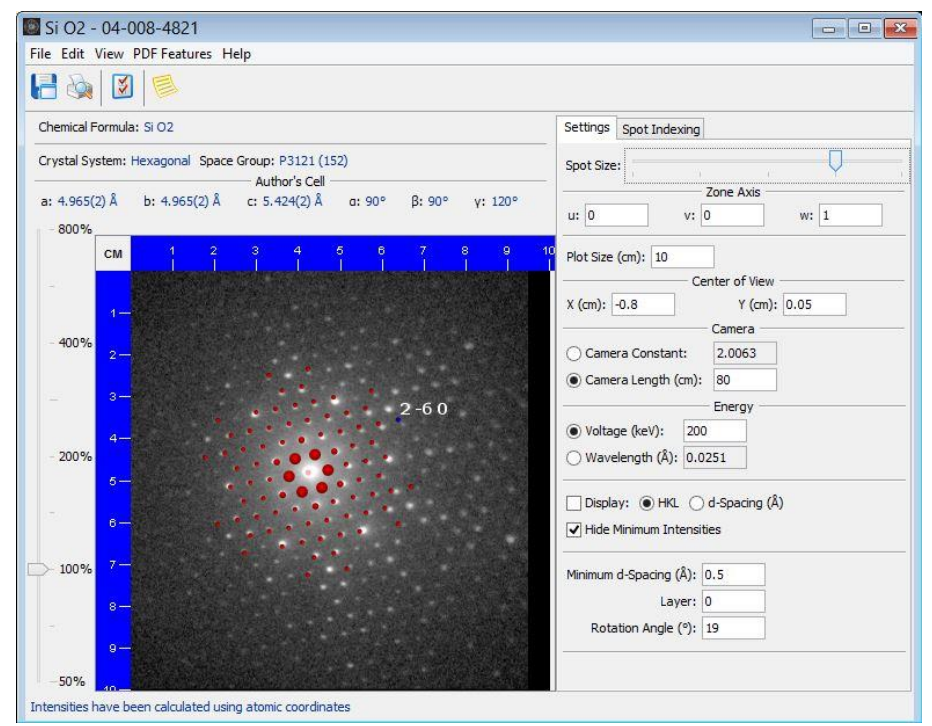

Figure 1. Users' experimental spot pattern of $\mathrm{SiO}_{2}$ (white spots) and graphical overlay of ED simulation of phase match from PDF-4+ (red spots).

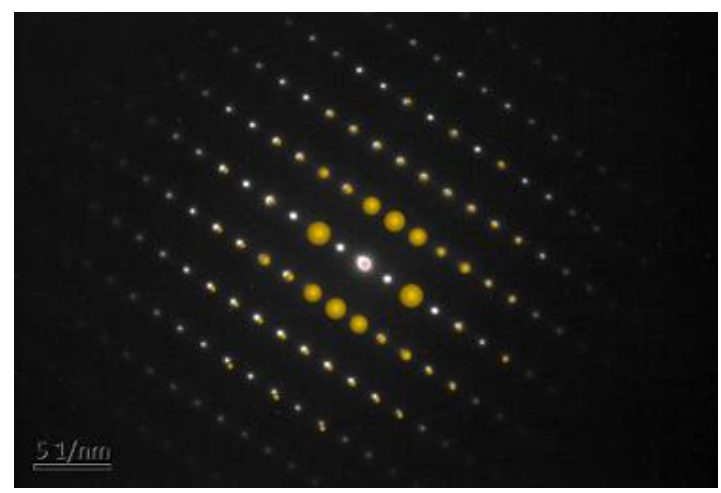

Figure 2. Users' experimental spot pattern of GaN (white spots) and graphical overlay of ED simulation of phase match from PDF-4+ (yellow spots).

Reference:

[1] Reid, J. et al., Microscopy Today, January (2011), p.38. 